\title{
The inhomogeneity of plastic deformation in ductile single crystals
}

\author{
Y W Zhang, T C Wang and N G Liang \\ LNM, Institute of Mechanics, Chinese Academy of Sciences, Beijing 100080, People's \\ Republic of China
}

Received 15 February 1994, accepted for publication 7 June 1994

\begin{abstract}
A discrete slip model which characterizes the inhomogeneity of material properties in ductile single crystals is proposed in this paper. Based on this model, rate-dependent finite element investigations are carried out which consider the finite deformation, finite rotation, latent hardening effect and elastic anisotropy. The calculation clearly exhibits the process from microscopic inhomogeneous and localized deformation to necking and the formation of LSBS and reveals several important features of shear localization. For example, the inhomogeneous deformation is influenced by the imperfections and initial non-uniformities of material properties. The inhomogeneous deformation may either induce necking which results in the lattice rotation and leads to geometrical softening, which in turn promotes the formation of CSBs, or induces heavily localized deformation. The microscopic localized deformation eventually develops into the LSBS and results in a failure. These results are in close agreement with experiment. Our calculations also find that the slip lines on the specimen's surface at necking become curved and also find that if the necking occurs before the formation of LSBS, this band must be misoriented from the operative slip systems. In this case, the formation of LSBS must involve non-crystallographuc effects. These can also be indirectly confirmed by experiment. All these suggest that our present discrete slip model offers a correct description of the inhomogeneous deformation characterization in ductile crystals.
\end{abstract}

\section{Introduction}

The inhomogeneity of plastic deformation is a common phenomenon in deformed crystalline solids, even in single crystals at the initial stage of plastic deformation. The inhomogeneous plastic deformation is concentrated into slip planes and slip bands, which vary in their participation in total deformation. It is usually the result of heterogeneous slip in certain slip systems.

Analysis of non-uniform and localized deformation in ductile single crystals have been carried out many times (Peirce et al [1,2], Nemat-Nasser [3]). They used crystal plasticity to model the non-uniform and localized deformation in ductile single crystals. The only imperfections considered in their calculations are the initial inhomogeneity of specimen's thickness or thermal softening. They considered the crystal as a homogeneous media. But as pointed by Neuhauser [4]; "slip lines are distributed randomly on the whole crystal length with slip line density depending on the strain'. The experimental results of Mader [5] and Zhang [6] also confirmed the discrete characterization of slip. It is also observed that only a small portion of crystallographic planes have taken part in the deformation and even much less taken part simultaneously at a time, i.e. only a limited number of sources has been active and each one for a limited time. Figure $1(a)$ and $(b)$ shows the randomly distributed slip lines on the specimen's surface which change with straining level. The pronounced 
hierarchy of slip heterogeneity depends on the ability of the dislocations destroying the obstacles. The ability for dislocations destroying the obstacles in a slip system, i.e. the critical resolved shear stress (CRSS) determines the distribution and the activation of slip lines.

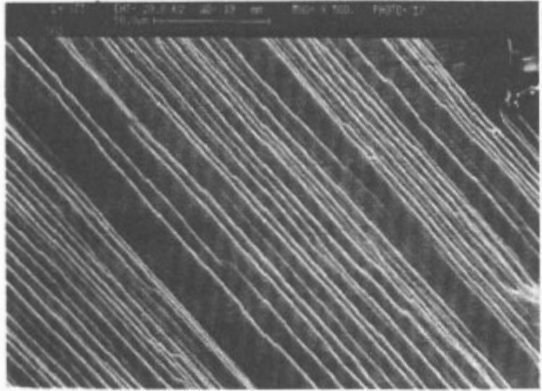

(a)

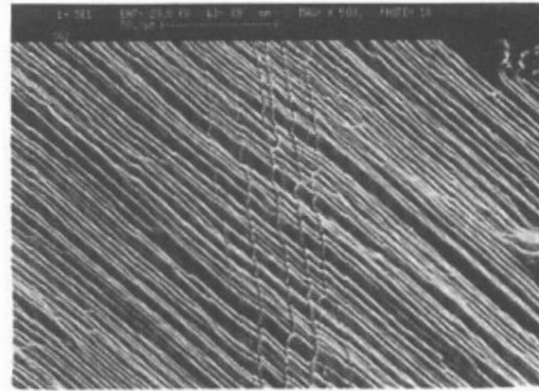

(b)

Figure 1. The morphology of slip lines on specimen's surface (Zhang [6]), (a) discrete slip lines at small deformation; (b) surface almost completely covered by the slip lines at large deformation.

The theoretical framework for analyzing the initiation of LSB was set up to associate the formulation of LSBs with material instability (Hill [7] and Asaro [8]). They employed the proposition that the localization is the result of a constitutive instability and is predictable from the pre-localized constitutive law. Based on the framework, Needlemann and Tvergaard [9] and Needleman [10] among others analyze the localized deformation. Their results are in close accord with the experimental observations for homogeneous or nearhomogeneous media.

It has been known that plastic deformation is inhomogeneous in the microscale even at the initial deformation stage, with continued straining, becomes mesoscopic inhomogeneous and generally through the onset of diffuse necking and/or LSBS. Failure usually ensues either by necking to a 'chisel edge' or by rupturing within intense shearing bands. Chang and Asaro [11] carried out experiments on localized shearing in FCC Al-Cu single crystals subject to several aging treatment. The precipitation hardened alloy containing coherent zones or incoherent and semicoherert precipitates form an interesting class of material that displays intense shearing band leading to fracture. Figure 2 shows an example of the formation of LSBs in Al-Cu single crystal. The crystal first deformed with randomly distributed fine slip lines on the surface, then underwent diffuse necking, the LSBs formed within the neck. Crystals that contained incoherent $\theta$ particles behaved similarly to those containing $\theta^{\prime}$ except that they underwent considerably more necking before the LSBs formed. In addition, the bands were not straight as shown in figure 3. Figure 3 shows a LSB in an Al-Cu crystal where curving of the band in a direction further away from the tensile axis is evident at specimen free edges. The localized shearing and necking were preceded by the formation of coarse slip bands (CSBs) as shown in figure 4(a) and (b). The inhomogeneous plastic deformation induced by the non-uniformity of matcrial properties results in the formation of CSBs which may then serve as imperfections in which the necking and LSBs set in. The figure also shows the LSBS become misoriented with respect to the CSBs (and the underlying crystallographic 
planes). If the LSBS are slipping on another slip plane as suggested by Elam [12] remains unresolved. The LSBS are not aligned with the operating slip plane as seen in figure $4(a)$. The curved slip lines at necking can be seen from figure $4(b)$. The curved slip lines may give us some insights about the shearing at necking, because the curved slip lines have similarity with the curved shape of LSBs. This also means that the lattice rotation plays an important role in the diffuse necking and formation of LSBS. The 'patchy' deformations were often observed in experiment (Piercy [13]) as shown in figure 5. Two reasons may be associated with this phenomenon, one is the latent hardening effect and the other is generated by the inhomogeneity of structural constituents in slip planes, e.g. by the change of the instability of barriers, the freeing of mobile dislocations from impurity atoms or alloying elements etc. The inhomogeneity of plastic deformation and its consequences have been proved to play an important role in mechanical behaviours (Kafka [14]). Because of the inhomogeneity of material property in single crystal, during deformation, each microvolume in crystal is differently strengthened. A difference inevitably appears in density of dislocations, and this results in even more inhomogeneous deformation.

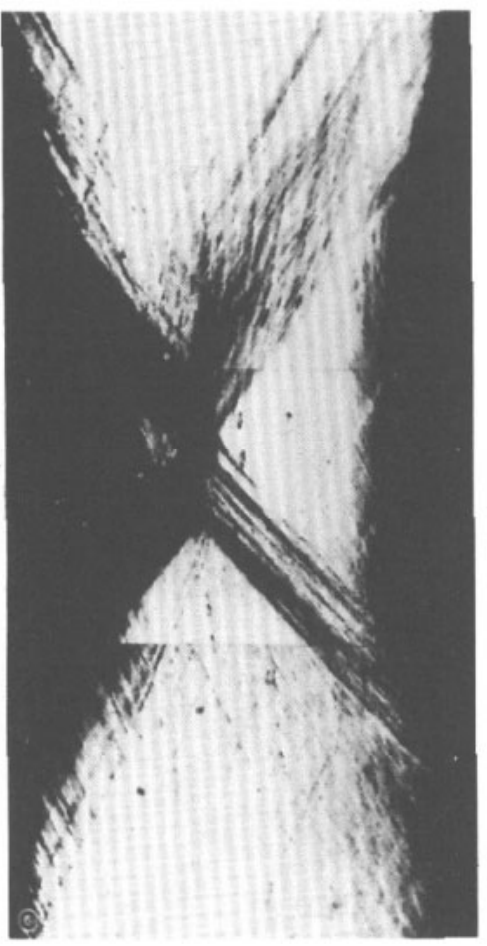

Figure 2. Localized shearing in a single crystal. Diffuse necking preceded shearing band formation. Curved slip lines can be observed (Chang and Asaro [11]).

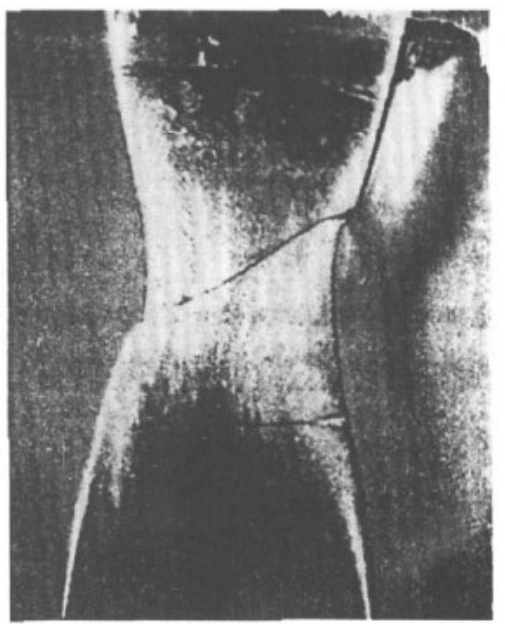

Figure 3. Localized shearing in single crystals. The shearing bands are somewhat curved especially near the free surfaces (Chang and Asaro [11]).

The experimental results with specimens made of the same material under the same conditions may exhibit some differences, e.g. the stress versus strain curves and the sites of necking and formation of LSBS may change. These variations may depend on the inhomogeneity of material properties which is randomly distributed in the specimen. Thus 

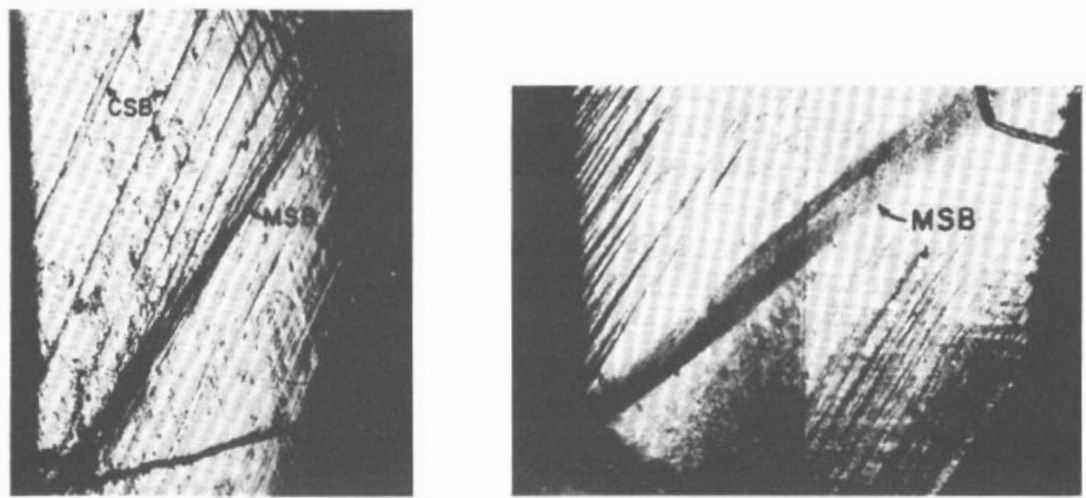

Figure 4. Localized shear bands (LSBs) and coarse slip bands (CSBS) in single crystals. Note the misorientation between the two kinds of bands especially evident in the left photo and also how the localized shearing bands form within clusters of csBs. Note also the curved slip lines (Chang and Asaro [1]]).
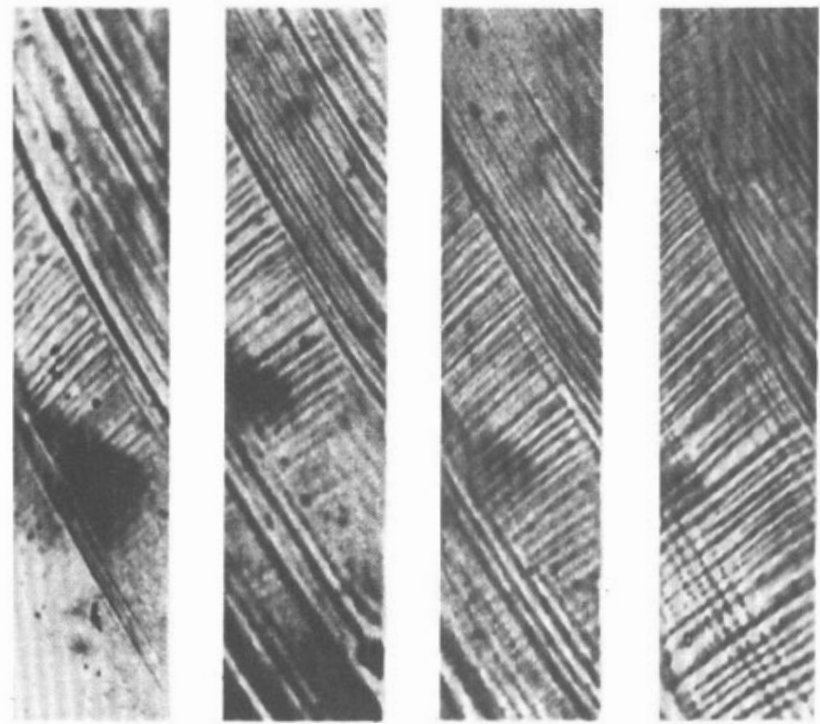

Figure 5. The motplology of 'patchy' slip pattern (Piercy et al [13]).

the statistical method based on the knowledge of the statistical description of microscopic properties may be used. The specimen can be divided into regions for which the crystal plasticity laws are in force. The microscopic parameters are considered as random variable. But most of them lack input information about the properties of microscopic regions and their distributions.

Based on the above analyses of micromechanism, we construct a discrete slip model which the inhomogeneity of material property is considered. By using this model, the inhomogeneous and localized deformation of ductile single crystals subject to tensile loading 
are analyzed numerically. The rate-dependent formulation is employed with precisely considering the finite deformation and finite lattice rotation. The latent hardening effect and elastic anisotropy are included in the calculation.

The crystallographic geometry and the presumed shearing modes in present paper are completely the same as that used by Rice [20] and Mohan et al [21]. The normal of specimen surface is taken to be [110] direction. This deformation mode is consistent with plane strain state. It is assumed that shear parallel to each slip plane involved simultaneous and equal amounts of slip along two face diagonal direction in that plane, so equal slip along [1 $\overline{1} 0]$ and $[0 \overline{1} 1]$ on (111) plane combine to produce an effective composite in-plane slip deformation on (111)[1 21$]$, similarly, [110] and [011] on (11) combine to produce (1i 1) [121] in-plane deformation. Finally the pair of (11i) [101] and (i11)[101] operating jointly result in-plane strain deformations. These kinds of combinations of slip systems are the only ones that can accommodate large plastic strains under plane strain conditions. As for plane strain deformation, the strain $\epsilon_{33}=\epsilon_{33}^{\mathrm{e}}+\epsilon_{22}^{\mathrm{p}}=0$. For the large in-plane plastic deformation, $\epsilon_{33}^{e}$ is much smaller and so is $\epsilon_{33}^{p}$ than the in-plane strain. So it is reasonable to ignore the slip systems that resulting in the plastic strain $\epsilon_{33}^{\mathrm{p}}$ entirely.

The calculation based on the discrete slip model clearly exhibits the process from locally inhomogeneous and localized deformation to necking and the formation of LSBS and reveals several important features of shear localization. For example, the inhomogeneous deformation is influenced by the imperfection and initial non-uniformities of material properties. The locally inhomogeneous deformation may either induce necking which results in the lattice rotation and leads to geometrical softening, this in turn promotes the formation of CSBs, or induces heavily localized deformation. The localized deformation eventually develops into the LSBS and results in a failure. These results are in close agreement with experiment. Our calculations also find that the slip lines on the specimen's surface at necking become curved and also find that if the necking occurs before the formation of LSBS, this band must be misoriented from the operative slip systems. In this case, the formation of LSBS must involve non-crystallographic effects. These also can be indirectly confirmed by experiment. This suggests that our present discrete slip model offers a correct description of the microscopic inhomogeneous_deformation characterization in ductile crystal.

The plan of the paper is as follows; in section 2 the discrete slip model and the constitutive law used in the calculation are described; in section 3 the numerical method is outlined and the results and discussions are presented; finally, some conclusions are given in section 4.

\section{Crystal model}

\subsection{The discrete slip model (DSM)}

The crystal usually deforms by two physically distinct mechanisms; (1) plastic deformation which consists of material shearing relative to a crystal lattice, and (2) elastic deformation of the lattice and material together. A significant difference between the crystal model used in previous researches (Taylor [15], Asaro [16]) and present models is that the DSM considers the spatial material heterogeneities as imperfection to reflect the influence of the microscopic inhomogeneity on the plastic deformation. Experiments show that the deformed crystal consists of many slip and slip free zones. The slip free zones are elastic bands and the slip zones are the slip bands as shown in figure $1(a)$ and $(b)$. Each slip band observed consists of fine slip lines which more or less densely packed in clusters with regular distance, with continued straining, the elastic bands gradually develop into slip bands. So the length 
scale for DSM is related to the width of slip band which depends the microstructure of materials. We suppose that whether the slip system is active or not depends on the critical resolved shear stress (CRSS) on this system.

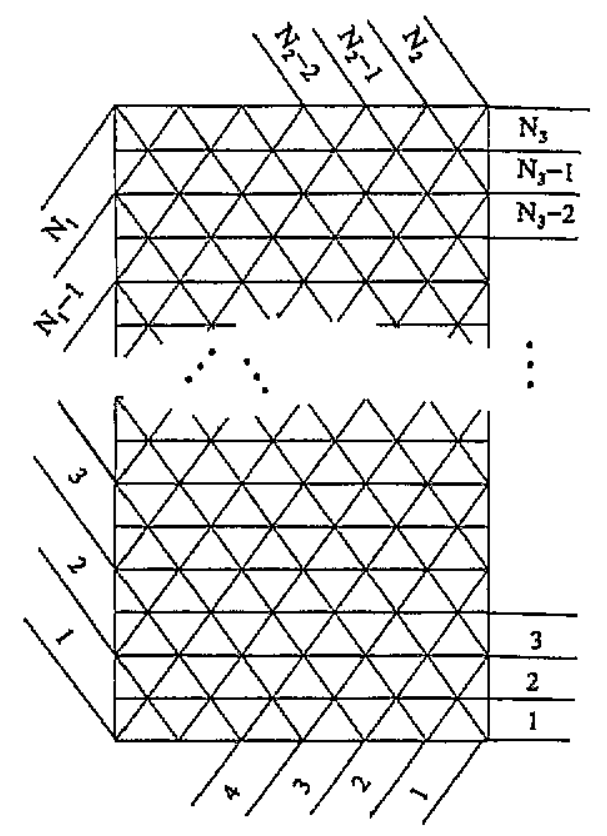

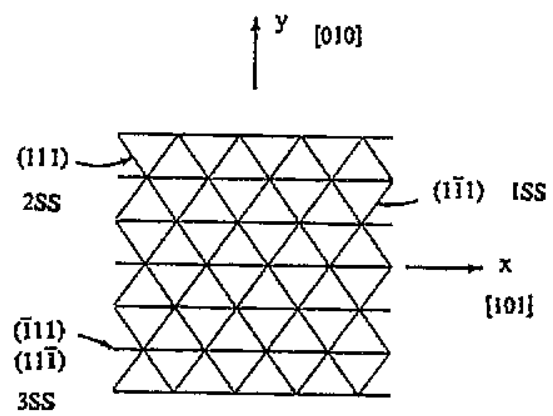

(b)

(a)

Figure 6. The discrete slip model (a) the arrangement of slip bands along three slip planes and numbering of the bands: $(b)$ the slip geometry.

Based on the above analysis, the DSM is constructed as follows: the crystal is divided into lots of bands in which the CRSS distributes randomly. Each band which is parallel to the slip planes is taken as crystal which the crystal constitutive laws are in force. The DSM for FCC crystal is shown in figure $6(a)$ and $(b)$. The normal of the crystal surface is $\langle 110\rangle$, the tensile axis $\langle 100\rangle$. The crystal is divided into three groups of bands, each is along particular shearing system (figure $6(a)$ ). The slip geometry is shown in figure $6(b)$.

In order to estimate the distribution of CRSS, an experiment should be designed to get this distribution. But at present this distribution is not available.

The physical meaning of this fluctuation is related with non-uniform distribution of initial dislocation density, the property of precipitate particles and the size and density of the particles etc. According to the working hardening theory [22], relation of the CRSS with the dislocation density has a form of $\tau_{\mathrm{CR}}=\tau_{\mathrm{PN}}+\alpha G b \rho^{0.5}, \tau_{\mathrm{PN}}$ is the Peierls-Nabarro stress, $\alpha$ material constant, $G$ is shear modulus, $b$ is the Burgers vector of the dislocations and $\rho$ the dislocation density. If we take $\rho$ as the initial dislocation density, and know the distribution of $\rho$, then the distributions of $\tau_{\mathrm{CR}}$ are known. The distributions of $\tau_{\mathrm{CR}}$ also greatly depend on misfit, size and interval of precipitates.

Here a phenomenological method is used to evaluate the non-uniform distributions. We observed that before the crystal yields, the slip lines already exist on the specimen's surface. When reaching the maxima of loading, almost the whole specimen's surface is full of the 


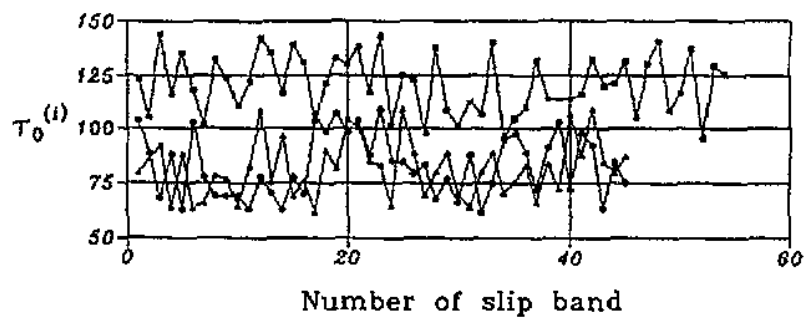

(a)

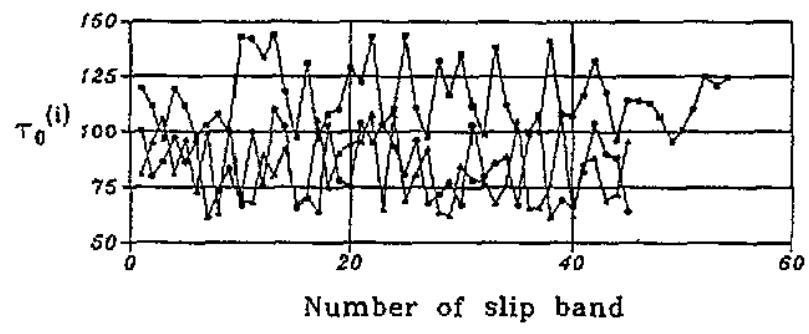

(b)

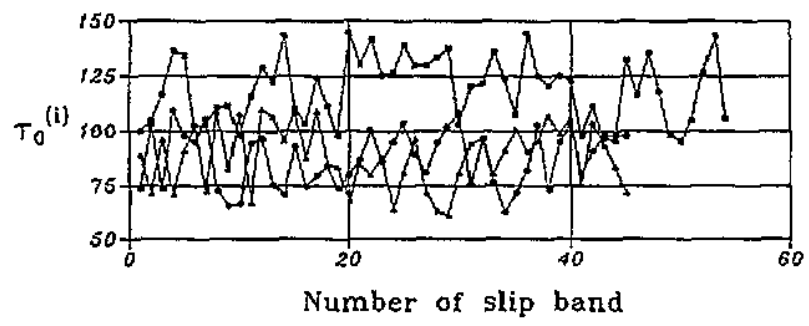

(c)

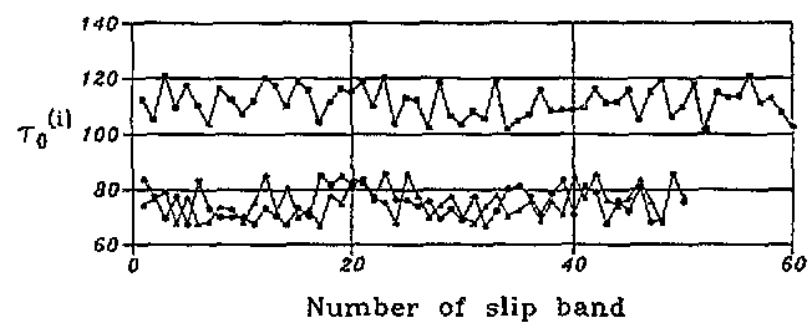

(d)

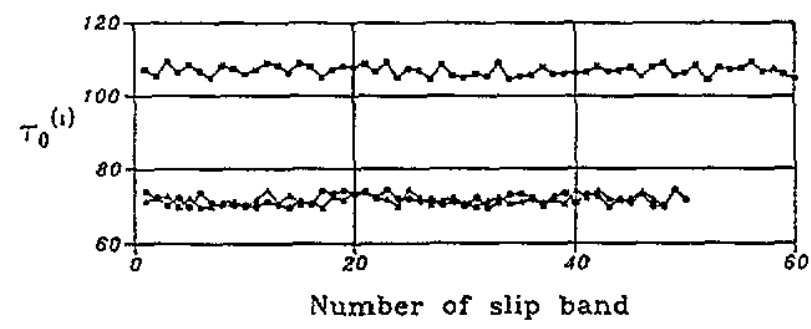

(e)

Figure 7 . The variations of critical shear stress $\tau_{0}^{(i)}$ with the number of slip band for three shearing systems (circle for (111)[12̄1] (denoted by 1SS), triangle for (1ī1)[121] (2SS) and square for $(11 \bar{i})[101]$ and $(\bar{i} 11)[101]$ operating jointly (3SS)). (a) for case 1 . (b) for case 2. (c) for case 3 . $(d)$ for case 4 . (e) for case 5 .

slip lines. Thus CRsS may change from $\tau_{0 L}$, the minimum critical resolved shear stress, to 


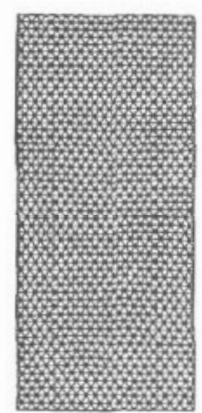

(a)

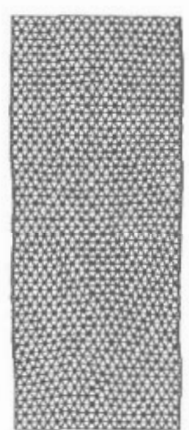

(b)

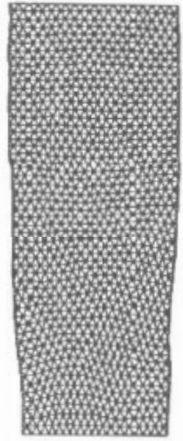

(c)

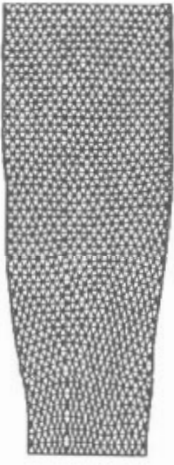

(d)

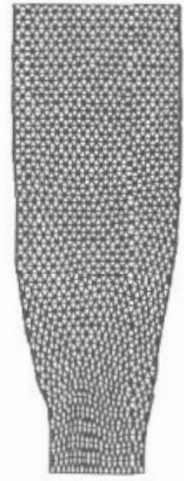

(e)

Figure 8. The deformed meshes for case 1 at $(a) U / L_{()}=0.05$. (b) $U / L_{0}=0.10$. (c) $U / S_{0}=0.15$. (d) $U / L_{0}-0.20$. (e) $U / L_{0}=0.25$

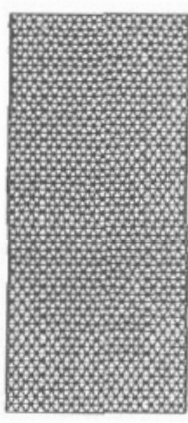

(a)

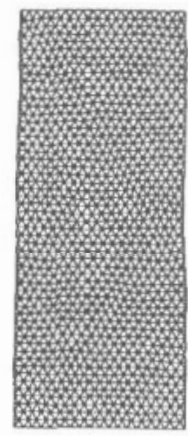

(b)

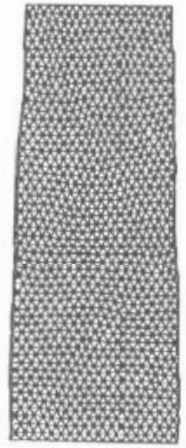

(c)

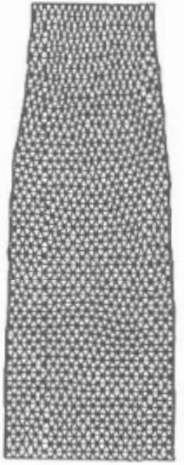

(d)

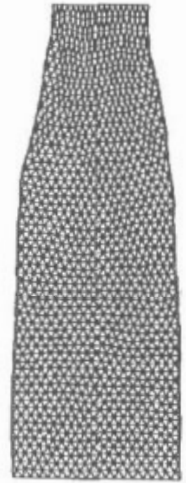

(e)

Figure 9. The deformed meshes for case 2 at (a) $U / L_{0}=0.05$. (b) $U / L_{0}=0.10$. (c) $U / L_{0}=0.15 .(d) U / L_{0}=0.20 .(e) U / L_{0}=0.25$

the $\tau_{0 \mathrm{U}}$, the maximum critical resolved shear stress in crystal, $\tau_{\mathrm{OL}}$ will be a little smaller than $\tau_{0}$, the critical resolved shear stress in crystal plasticity $[1,2] . \tau_{0 \mathrm{U}}$ will be greater than $\tau_{0}$, but smaller than $\tau_{s}$, the saturated resolved shear stress $[1,2]$. Thus, the following distribution is employed

$$
\tau_{0}^{(\mathrm{i})}=\alpha \tau_{0}+\tau_{\mathrm{am}}\left(R^{(\mathrm{i})}-\beta\right) .
$$

In FCC crystal, for (11) $[121]$ and $(111)[1 \overline{2} 1]$ shearing systems, $\alpha=2 / \sqrt{3}$, for the pait of systems $(11 \overline{1})[101]$ and (111) [101] operating jointly, $\alpha=\sqrt{3} . \tau_{\text {am }}$ is the amplitude of CRSS, and $R^{(i)}$ is the random variable changing from 0 to 1 . The $R^{(i)}$ is generated by a random method, $\beta$ is a constant which determines the mean value of CRSS.

The inhomogeneous microstructures also result in the fluctuations of the saturation shear stress $\tau_{\mathrm{s}}$, the initial hardening moduli $h_{0}$ and the saturation hardening moduli $h_{\mathrm{s}}$. Including all these fluctuations will make the problem more complicated. Here, for simplicity, only 


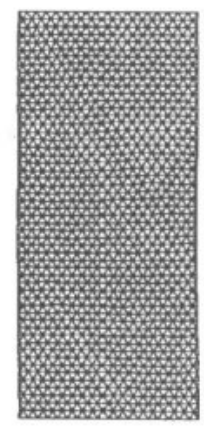

(a)

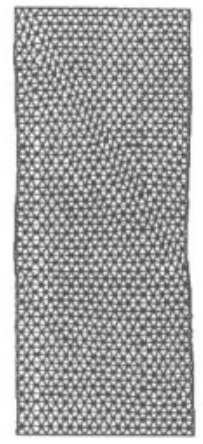

(b)

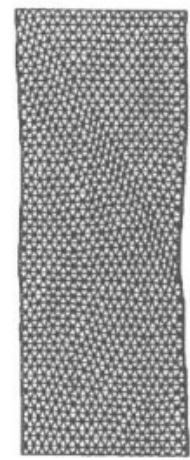

(c)

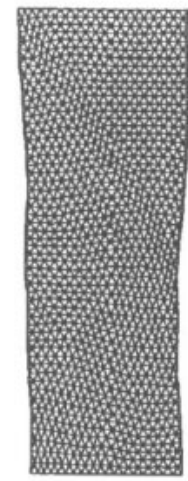

(d)

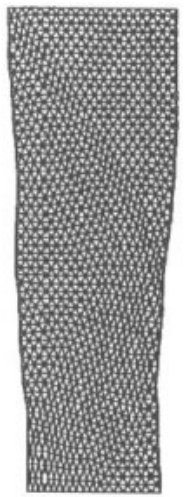

(e)

Figure 10. The deformed meshes for case 3 at (a) $U / L_{0}=0.05$, (b) $U / L_{0}=0.10$. (c) $U / L_{0}=0.15$. (d) $U / L_{0}=0.20$. (e) $U / L_{0}=0.25$.

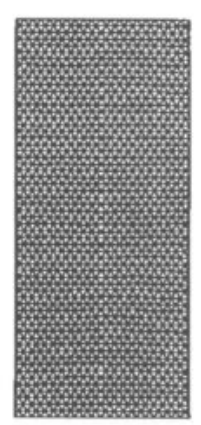

(a)

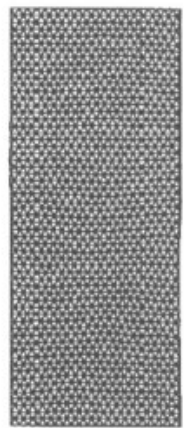

(b)

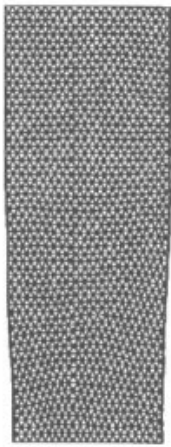

(c)

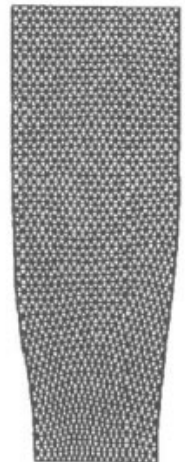

(d)

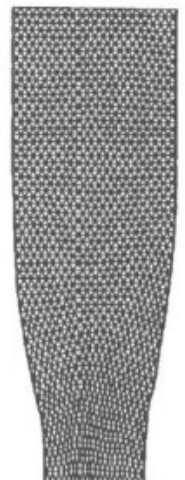

(e)

Figure 11. The deformed meshes for case 4 at (a) $U / L_{0}=0.05$. (b) $U / L_{0}=0.10$. (c) $U / L_{0}=0.15$. (d) $U / L_{0}=0.20$. (e) $U / L_{0}=0.25$.

the fluctuation of CRSS is considered. Further investigations including all these fluctuations are our future research work.

The DSM essentially represents the slipping crystal with microscopically spatial heterogeneities which describe the inhomogeneity of mobile dislocation distribution and the ability of dislocation escaping from precipitates atom and blocking. The inhomogeneous plastic deformation can be simulated based on the DSM.

We use this model to examine the local inhomogeneous plastic deformation and its effect on the LSB and necking. It should be noted that, for the homogeneous or near-homogeneous crystal with fine precipitates, the slip lines are fine and relatively uniform, the continuous slip model (Peirce et al [2] and Needleman et al [18]) can give very good results. Here we consider the crystals containing large precipitates with large interval. Their slip bands distribute non-uniformly. The physical meaning of the width scale of the distributions corresponds to width of the slip bands. Our calculations reveal some important relations between the microscopically spatial material heterogeneity and plastic deformation pattern. 


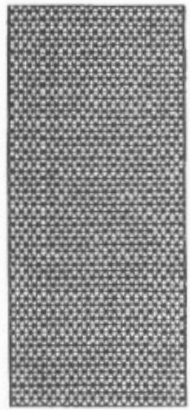

(a)

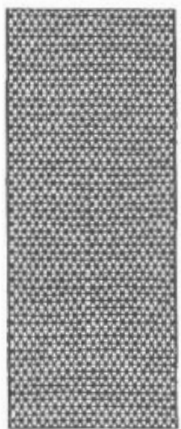

(b)

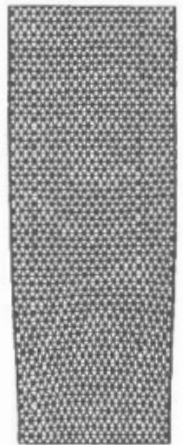

(c)

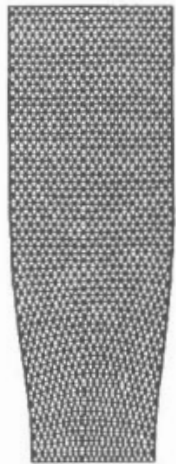

(d)

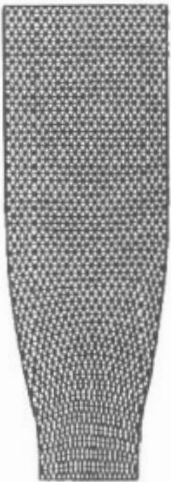

(c)

Figure 12. The deformed meshes for case 5 at (a) $U / L_{0}=0.05$. (b) $U / L_{0}=0.10$. (c) $U / L_{13}=0.15$. (d) $U / L_{0}=0.20$. (e) $U / L_{0}=0.25$

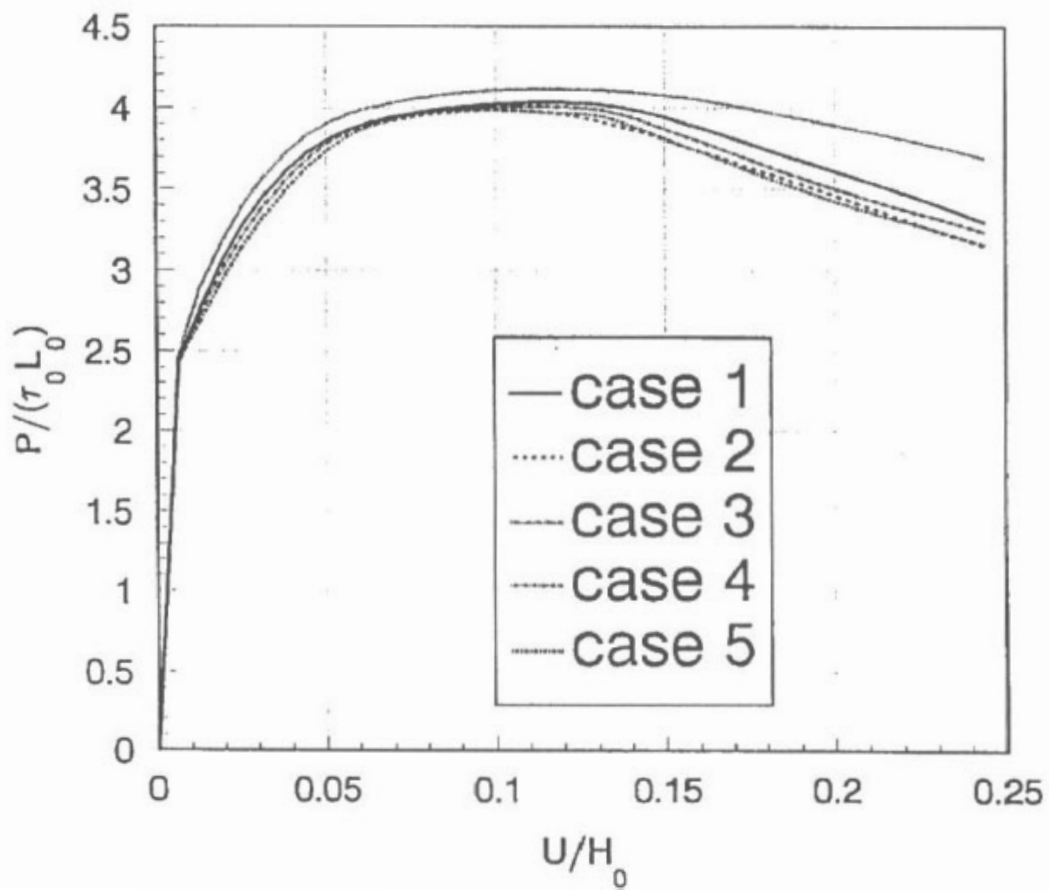

Figure 13. Load versus engineering strain curves for the five cases.

\subsection{Constitutive relation}

The crystal plasticity for describing plastic deformation of crystalline solids at a full finite deformation constitutive formulation is given by Hill and Rice [17] and Asaro [16], the crystal constitutive formulation here is taken from Peirce et al [2] and Needleman et al [18]. 


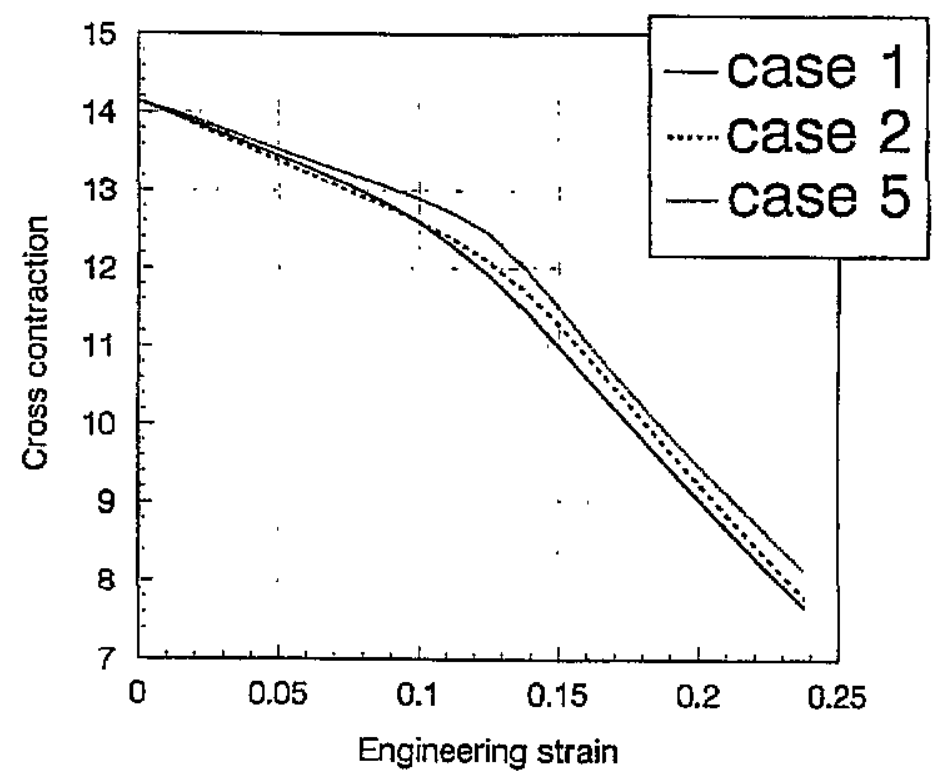

Figure 14. The variations of cross contraction with engineering strain for case 1 , case 2 and case 5.

The deformation gradient $F$ is written as

$$
F=F^{*} \cdot F^{\mathrm{p}}
$$

where $F^{*}$ arises from the stretching and rotation of the crystal lattice and $F^{\mathrm{P}}$ is the deformation due to plastic slipping.

In the deformed lattice, the slip direction and the normal to the slip plane are given by $[1,2]$

$$
s^{*(\alpha)}=F^{*} \cdot s^{(\alpha)} \quad m^{*(\alpha)}=m^{(\alpha)} \cdot\left(F^{*}\right)^{-1} .
$$

For each slip system, symmetrical and anti-symmetrical tensors are defined by

$$
\begin{aligned}
& P^{(\alpha)}=\frac{1}{2}\left(s^{*(\alpha)} \otimes m^{*(\alpha)}+m^{*(\alpha)} \otimes s^{*(\alpha)}\right) \\
& W^{(\alpha)}=\frac{1}{2}\left(s^{*(\alpha)} \otimes m^{*(\alpha)}-m^{*(\alpha)} \otimes s^{*(\alpha)}\right) .
\end{aligned}
$$

Then, in the deformed crystals, the plastic deformation rate and plastic spin rate can be written as

$$
D^{\mathrm{p}}=\sum_{\alpha} p^{(\alpha)} \dot{\gamma}^{(\alpha)} \quad \Omega^{\mathrm{p}}=\sum_{\alpha} W^{(\alpha)} \dot{\gamma}^{(\alpha)}
$$

where $\dot{\gamma}^{(\alpha)}$ denotes the shearing rate on the $\alpha$ th slip system.

$$
B^{(\alpha)}=j\left(W^{(\alpha)} \cdot \sigma-\sigma \cdot W^{(\alpha)}\right)
$$


(a)

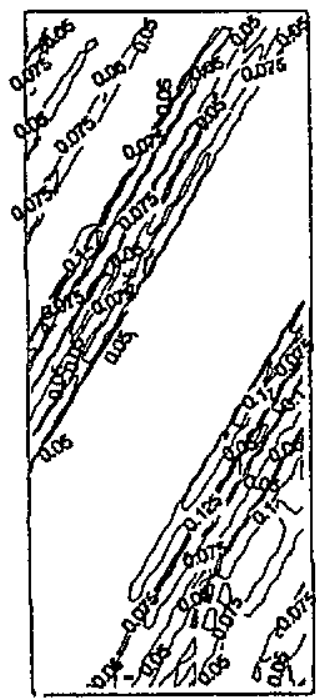

(d)

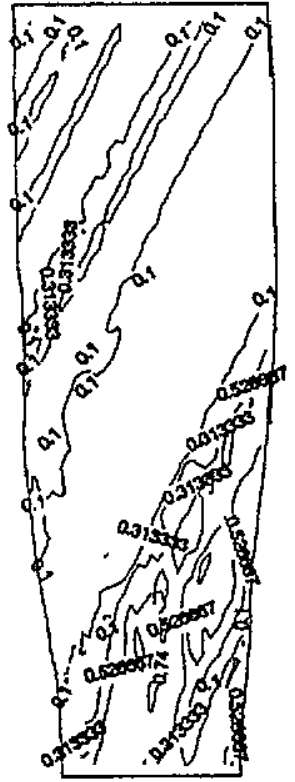

(b)

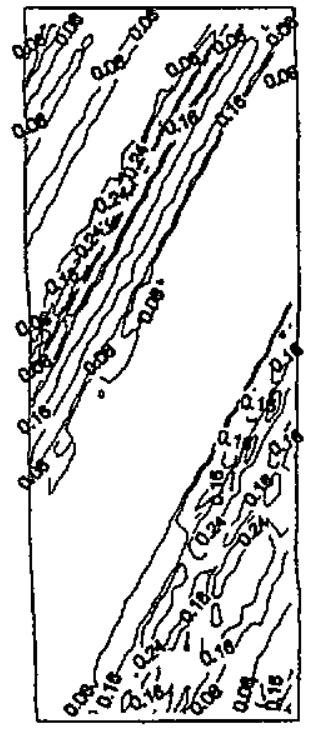

(c)

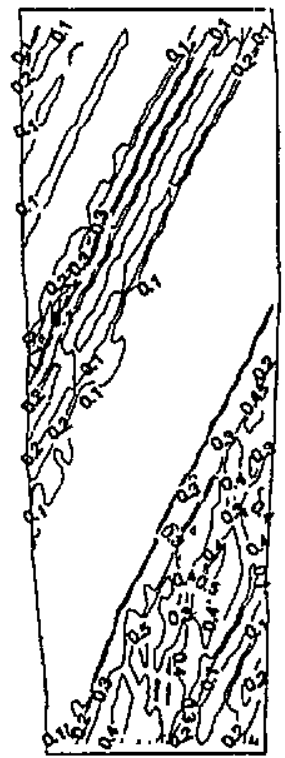

(e)

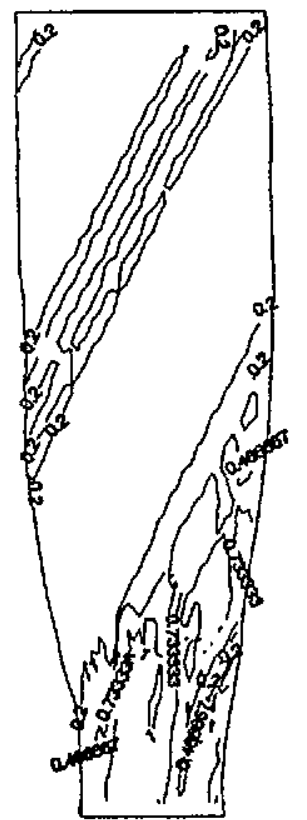

Figure 15. The contour plots of 1 SS shear strain for case 1 (a) $U / L_{0}=0.05$. (b) $U / L_{0}=0.10$. (c) $U / L_{0}=0.15$. (d) $U / L_{0}=0.20$. (e) $U / L_{0}=0.25$.

the crystal constitutive equation can be written:

$$
\dot{S}=\left(L^{\circ}+(j-1) \Gamma\right]: \dot{E}-\sum_{\alpha}\left[\left(L^{\circ}+j \Gamma\right): P^{(\alpha)}+B^{(\alpha)}\right] \dot{\gamma}^{(\alpha)} .
$$

The elastic moduli $L^{\circ}$ is taken to be orthogonal anisotropic, and convects with the lattice deformation $F^{*}$, i.e. 
(a)

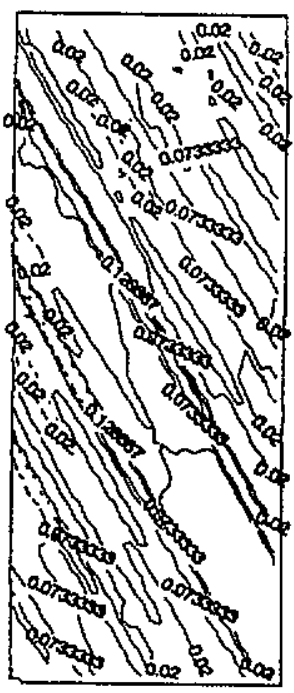

(d)

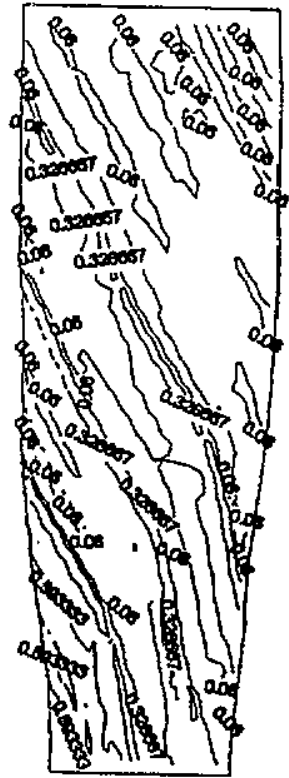

(b)

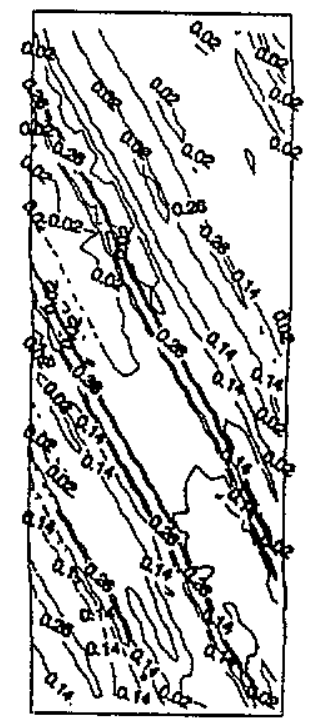

(c)

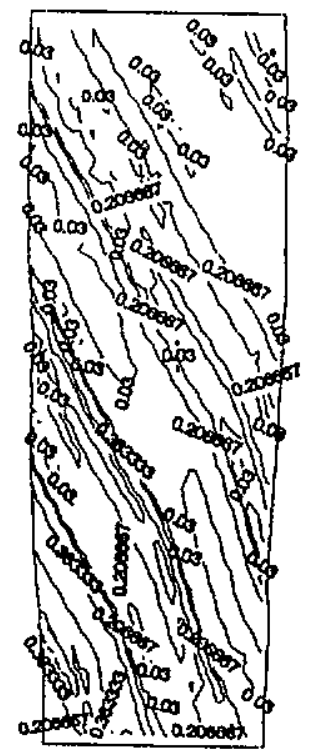

(e)

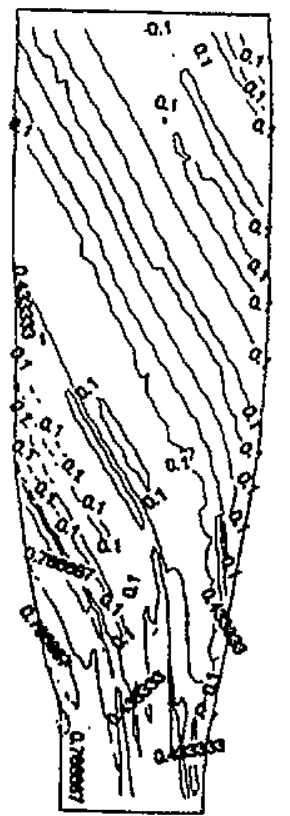

Figure 16. The contour plots of 2SS shear strain for case 1 at $(a) U / L_{0}=0.05$. (b) $U / L_{0}=0.10$. (c) $U / L_{0}=0.15$. (d) $U / L_{0}=0.20$. (e) $U / L_{0}=0.25$.

$$
L^{\circ}=F^{*} \cdot F^{*} \cdot L \cdot F^{* T} \cdot F^{* T}
$$

where $L$ is the elastic moduli at initial undeformed state and $\Gamma$ is the fourth-order tensor containing the stress terms $j\left(\sigma_{i l} \delta_{j k}+\sigma_{i k} \delta_{j l}\right), j=\operatorname{det}(F), \sigma$ is the Euler stress. It is needed to transfer the $L$ to the elastic moduli in (110) plane for present calculation.

In the present formulation, each $\dot{\gamma}^{(\alpha)}$ is dependent on the dislocation driving force and current internal structure. The expression used here for the shearing rate is of the power 
(a)

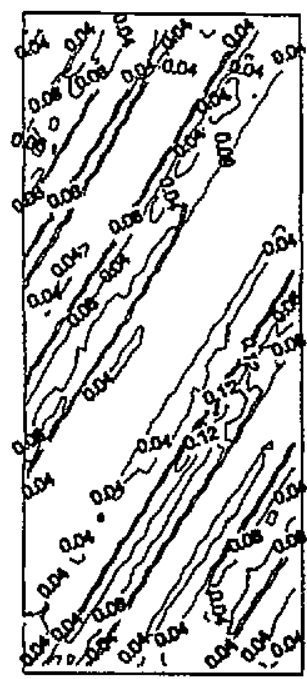

(d)

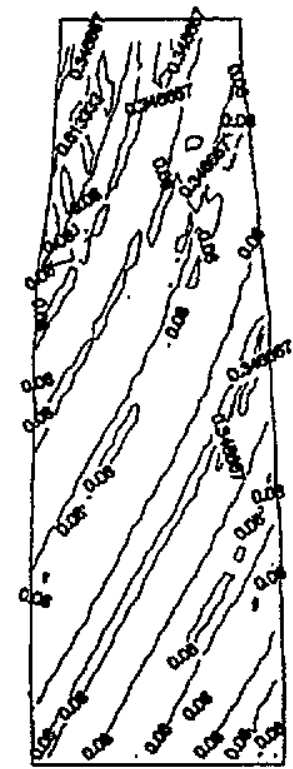

(b)

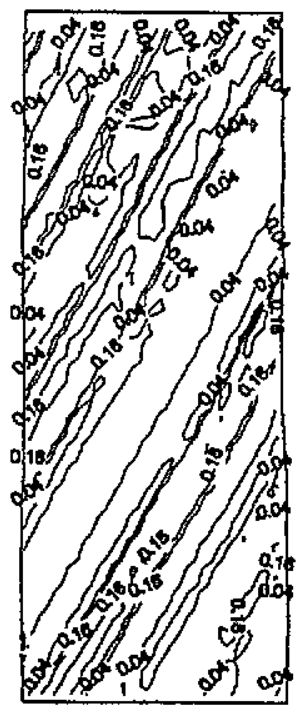

(c)

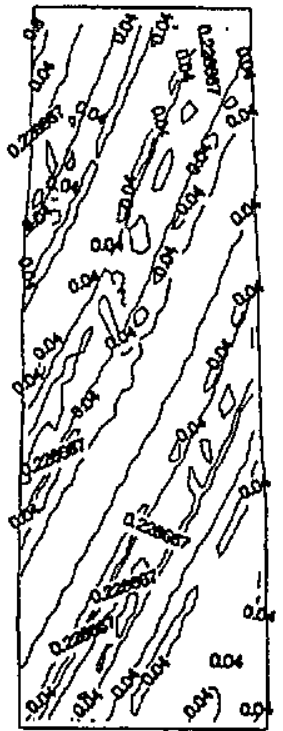

(e)

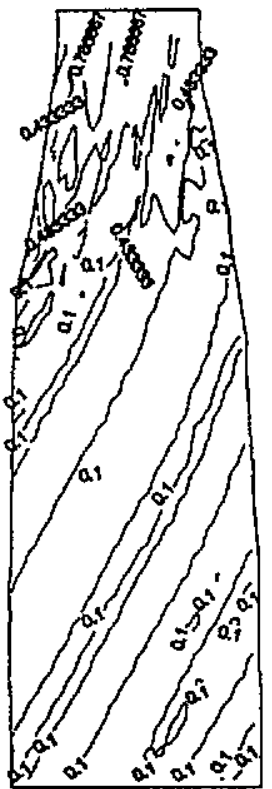

Figure 17. The contour plots of 1SS shear strain for case 2 at (a) $U / L_{0}=0.05$. (b) $U / L_{0}=0.10$. (c) $U / L_{0}=0.015$. (d) $U / L_{0}=0.20$. (e) $U / L_{0}=0.25$.

law form used in $[2,18]$

$$
\dot{\gamma}^{(\alpha)}=\dot{a}^{(\alpha)}\left[\frac{\tau^{(\alpha)}}{g^{(\alpha)}}\right]\left[\left|\frac{\tau^{(\alpha)}}{g^{(\alpha)}}\right|\right]^{(1 / m)-1}
$$

where $\tau^{(\alpha)}$ is the resolved shear stress, $g^{(\alpha)}$ is the current strain hardened state function on the $\alpha$ th slip system, exponent $1 / m$ characterizes the material rate sensitivity. $\dot{a}^{\alpha}$ is the reference shearing rate on $\alpha$ th slip system. 
(a)
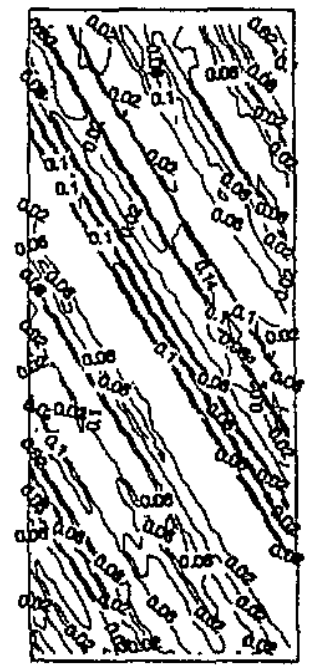

(d)

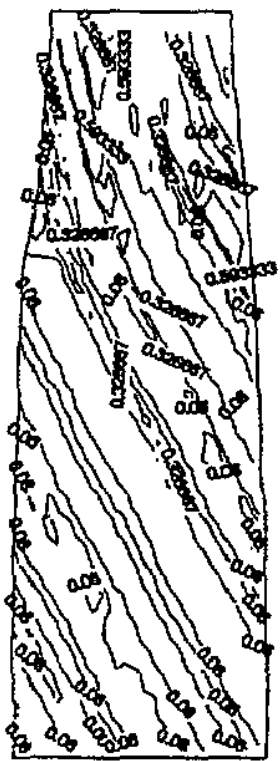

(b)

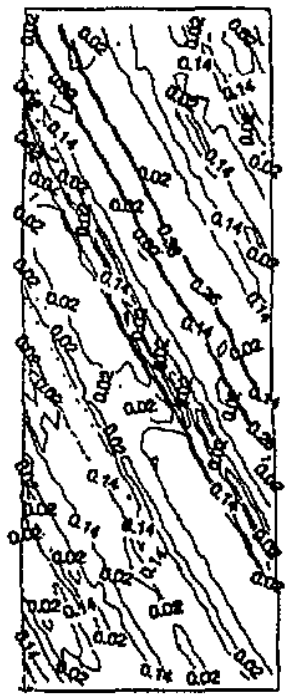

(c)

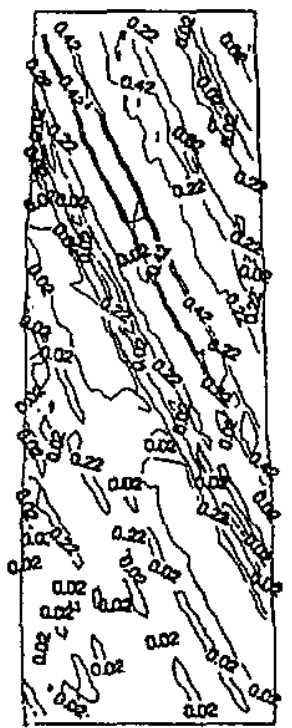

(e)

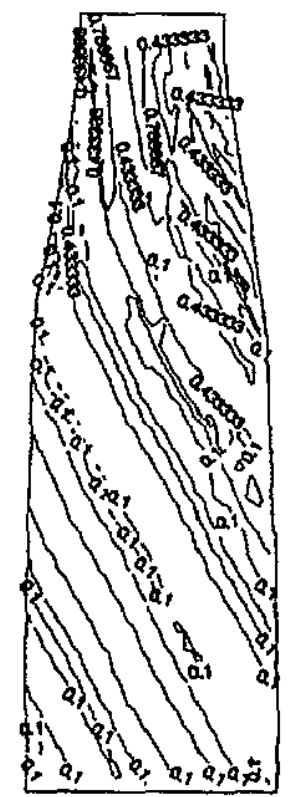

Figure 18. The contour plots of $2 S S$ shear strain for case 2 at (a) $U / L_{0}=0.05$. (b) $U / L_{0}=0.10$. (c) $U / L_{0}=0.15$. (d) $U / L_{0}=0.20$. (e) $U / L_{0}=0.25$.

The evolution of the function $g^{(\alpha)}$ is specified by

$$
\dot{g}^{(\alpha)}=\sum_{\beta} h_{\alpha \beta}\left|\dot{\gamma}^{(\beta)}\right|
$$

where $h_{\alpha \beta}$ is the hardening moduli, and can be expressed by

$$
h_{\alpha \beta}=h(\gamma)\left[q+(1-q) \delta_{\alpha \beta}\right]
$$


(a)

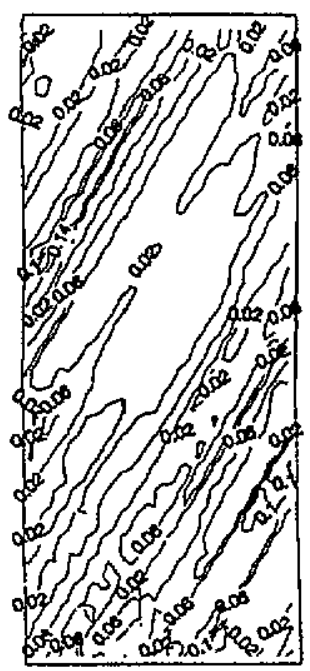

(d)

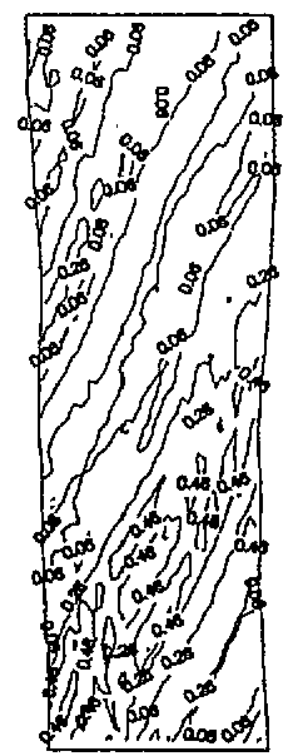

(b)

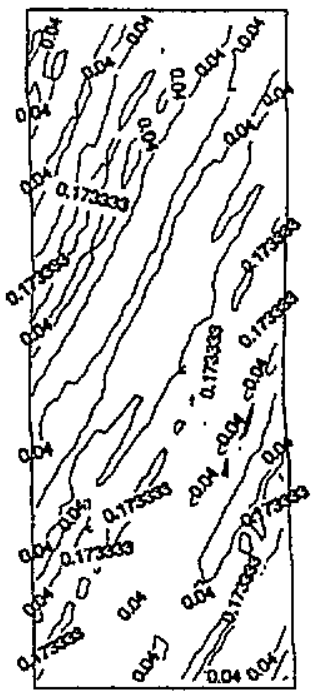

(c)

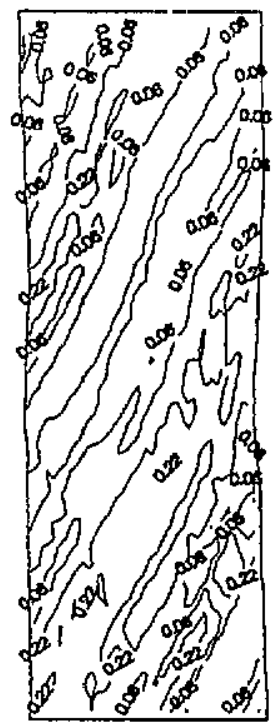

(e)

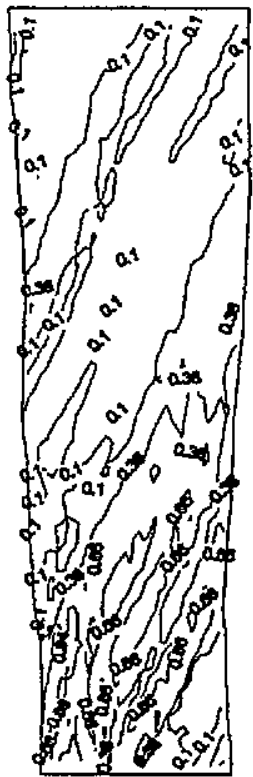

Figure 19. The contour plots of $1 S S$ shear strain for case 3 at $(a) U / L_{0}=0.05$. (b) $U / L_{0}=0.10$. (c) $U / L_{0}=0.15$. (d) $U / L_{0}=0.20$. (e) $U / L_{0}=0.25$.

here, parameter $q$ sets the levels of latent hardening as compared to the 'self-hardening' of the slip systems.

$h(\gamma)$ is specified by [23]

$$
h(\gamma)=h_{\mathrm{s}}+\left(h_{0}-h_{\mathrm{s}}\right) \operatorname{sech}^{2}\left[\frac{\left(h_{0}-h_{\mathrm{s}}\right) \gamma}{\tau_{\mathrm{s}}-\tau_{0}}\right]
$$

where 
(a)

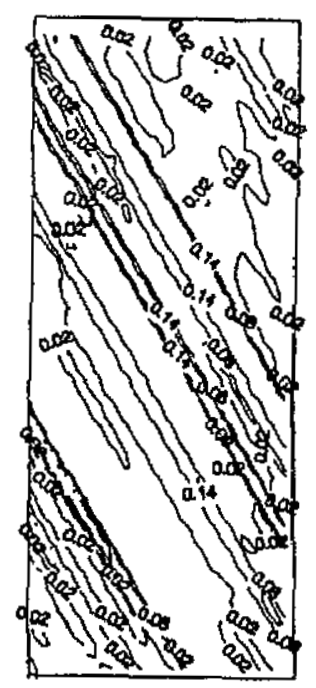

(b)

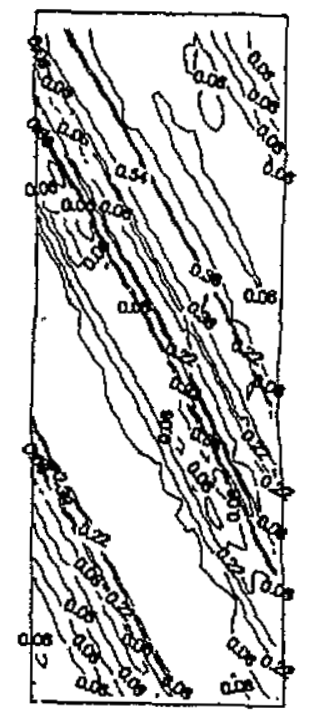

(c)

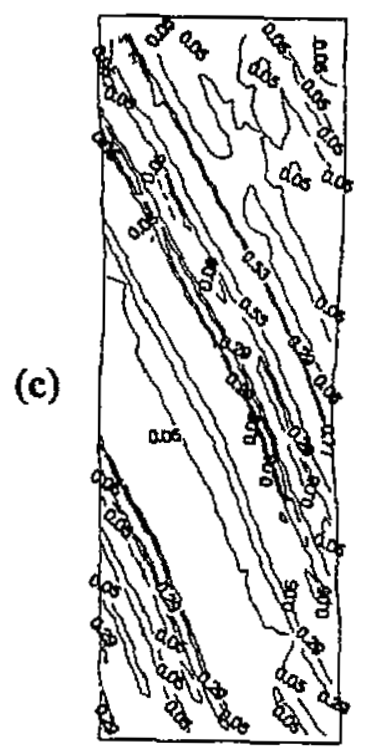

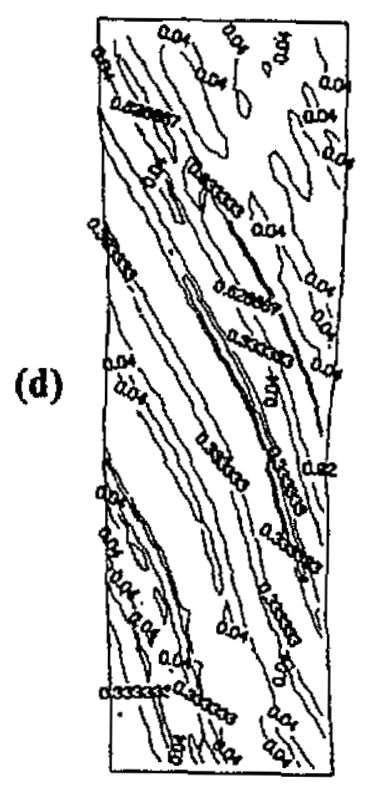

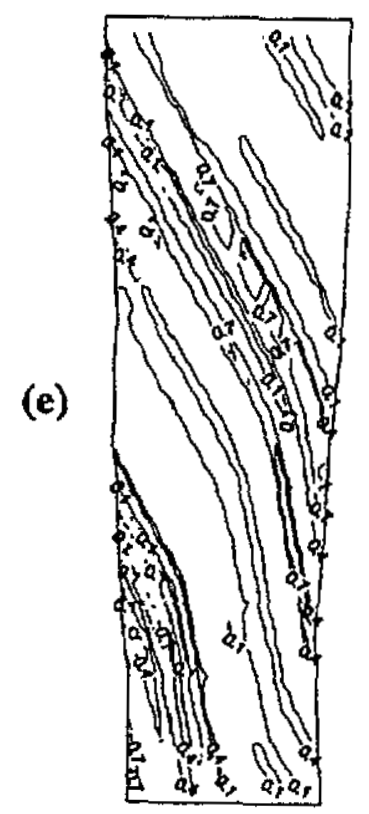

Figure 20. The contour plots of $25 S$ shear strain for case 3 at $(a) U / L_{0}=0.05$. (b) $U / L_{0}=0.10$. (c) $U / L_{0}=0.15$. (d) $U / L_{0}=0.20$. (e) $U / L_{0}=0.25$.

$$
\gamma=\sum_{\alpha}\left|\gamma^{(\alpha)}\right|
$$

and $\tau_{s}$ denotes a saturation strength $h_{0}$ represents an initial hardening moduli, $\tau_{0}$ is initial critical resolved shear stress, $h_{\mathrm{s}}$ is the saturation hardening moduli. 


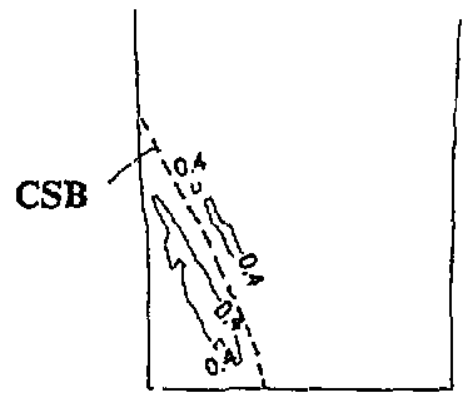

(a)

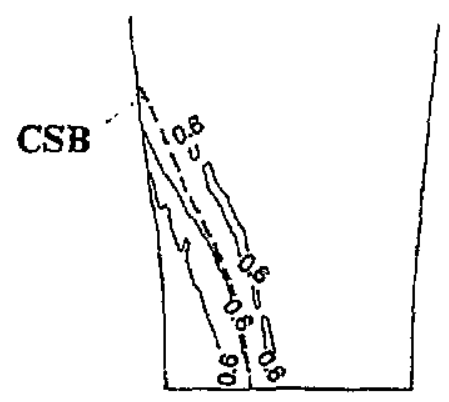

(b)

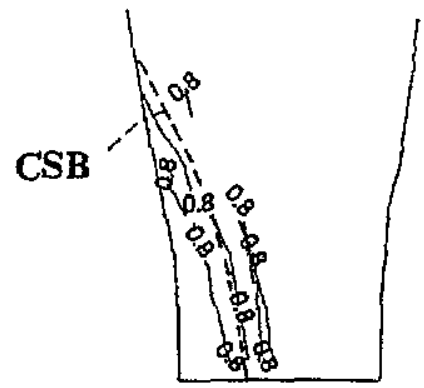

(c)

Figure 21. The contour plots of localized shearing bands together with the slipping band (dashed line) for case 4. (a) $U / L_{0}=0.15$. (b) $U / L_{0}=0.20$. (c) $U / L_{0}=0.25$. Note that the misorientation between the two kinds of bands.

\section{Numerical calculations and discussions}

\subsection{Numerical method}

The updated Lagrange method is adopted in the analysis with symmetrical Kirchhoff stress $S$-Green strain $\mathrm{E}$ formulation. Taking $t=t_{\mathrm{N}}$ as the reference configuration, using $S_{(\mathbb{N})}=\sigma$, $\Delta S_{(\mathrm{N})}=\dot{S} \Delta t, \Delta E_{(\mathrm{N})}=\dot{E} \Delta t$. Thus the virtual work incremental principle can be expressed by (no body force is considered)

$$
\begin{gathered}
\int_{V(N)} \Delta S_{(N) k l} \delta \frac{\partial \Delta u_{k}}{\partial x_{l}} \mathrm{~d} V+\int_{V(N)} \sigma_{k l} \frac{\partial \Delta u_{m}}{\partial x_{l}} \delta \frac{\partial \Delta u_{m}}{\partial x_{k}} \mathrm{~d} V-\int_{A(N)} \Delta T_{k} \delta \Delta u_{k} \mathrm{~d} A \\
=\int_{A(N)} T_{k} \delta \Delta u_{k} \mathrm{~d} A-\int_{V(N)} \sigma_{k l} \delta \frac{\partial \Delta u_{k}}{\partial x_{l}} \mathrm{~d} V .
\end{gathered}
$$

Employed (7) in conjunction with (13) leads to an extremely small time step to ensure numerical stability. In order to increase the stable time step, so-called rate tangent algorithm $[2,18]$ is employed in present calculation. This formulation gives a tangentmodulus method for solving the governing rate equation. The method is explicit in that no iterations are required. Once the displacement rate has been determined by solving the rate 

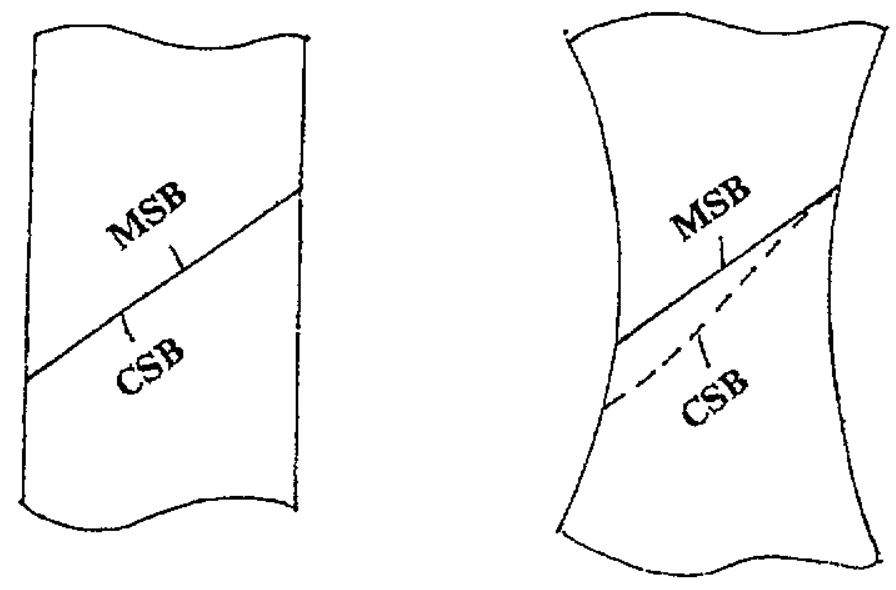

(a)

(b)

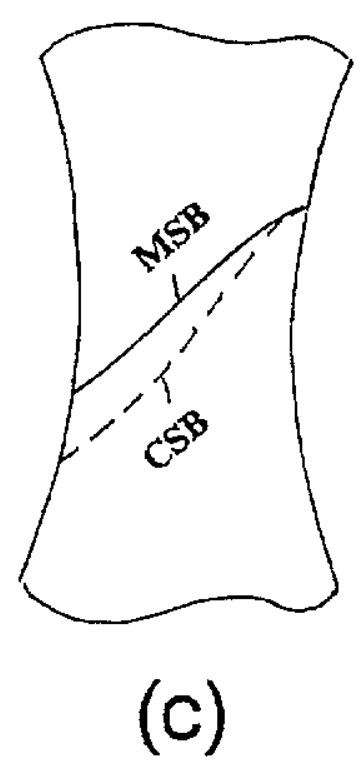

Figure 22. The relation between the formation of LSB and CSB. Note that the lattice rotations due to necking play an important role. Note also that non-crystallographic effect involved in (b) and $(c)$.

boundary-value problem, updating takes place using linear incremental procedure: $F\left(t_{N+1}\right)$ is computed directly as $F\left(t_{N}\right)+\Delta t \dot{F}, F^{\mathrm{p}}\left(t_{N+1}\right)$ is updated by $\left(\sum_{\alpha} s^{(\alpha)} \otimes m^{(\alpha)} \dot{\gamma}^{(\alpha)}\right) \cdot F^{\mathrm{p}} \Delta t$, then $F^{*}\left(t_{N+1}\right)$ is calculated as $F\left(t_{N+1}\right)\left(F^{\mathrm{P}}\left(t_{N+1}\right)\right)^{-1}$, using $(7), S$ can be calculated, and $S_{(N)}\left(t_{N+1}\right)=\sigma+\Delta t \dot{S}$, according to the stress transforming relation, $\sigma\left(t_{(N+1)}\right)$ can be obtained. When the internal variables have all been updated, a new current state is defined, and the process repeated.

Consideration is restricted to tensile deformation by prescribing the end-displacement 
rate. The tensile axis is aligned with $y$-direction. The boundary conditions on the calculated model analyzed numerically are:

$\begin{array}{llll}\dot{T}_{x}=0 & \dot{T}_{y}=0 & \text { at } x=0 & y \neq 0 \\ \dot{T}_{x}=0 & \dot{T}_{y}=0 & \text { at } x=L_{0} & \\ \dot{u}_{x}=0 & & \text { at } x=0 & y=0 \\ \dot{u}_{y}=0 & \dot{T}_{x}=0 & \text { at } y=0 & \\ \dot{u}_{y}=\dot{U} & \dot{T}_{x}=0 & \text { at } y=H_{0} . & \end{array}$

\subsection{Numerical results and discussions}

The finite element computation using DSM are carried out to analyze the five cases with different inhomogeneities of CRSS. In each case, the following original data are used in calculation: $C_{11}=842 \tau_{0}, C_{12}=607 \tau_{0}, C_{44}=377 \tau_{0}, \dot{a}=0.001, m=0.005, q=1.4$, $h_{0}=8.9 \tau_{0}, \tau_{s}=1.8 \tau_{0}, h_{\mathrm{s}}=0$. The values of material constants are typical of Al-Cu alloys $[11,19]$. The crystal geometry is specified by $L_{0} / H_{0}=\sqrt{2} / 3$.

In equation (1), $\tau_{0}=60.84 \mathrm{MPa}, \tau_{\mathrm{am}}=50 \mathrm{MPa}$ for case 1,2 and $3, \tau_{\mathrm{am}}=20 \mathrm{MPa}$ for case $4, \tau_{\mathrm{am}}=5 \mathrm{MPa}$ for case $5, \beta=0.2, R^{(i)}$ is chosen as a random variable. The $R^{(i)}$ s, $(i$ is the number of discrete slip bands), along (111) [121], along (111)[1211] and combination of (111)[101] and (11) [101] for five cases are shown in figures $7(a)-(e)$.

As for the intervals of the slip bands are usually several microns, using DSM to calculate the macroscopic specimen requires large computer time. In the present paper, only mesoscopic specimens are used in the calculation, where $L_{0}=90 \mu \mathrm{m}, H_{0}=$ $270 / \sqrt{2} \mu \mathrm{m}$. Thus the effects of the discrete and inhomogeneous slip bands on the mesoscopic deformation are reported here.

The finite element grids in the numerical investigations are based on constant-strain triangular elements. The number of finite element discretizations is 2072 for case 1,2 and 3 , and 2460 for case 4 and 5 .

The deformed meshes at $U / H_{0}=0.05,0.1,0.15,0.20,0.25$ for five cases are shown in figures 8(a)-(e)-figures 12(a)-(e). Except for case 3, the other four cases show a similar deformation mode, i.e. when $U / H_{0}<0.10$, the deformation pattern mesoscopically exhibits uniform mode. Only slightest hints of imperfection at specimen boundary and of microscopic inhomogeneity of slip deformation are observed. With continued straining, the microscopic inhomogeneity gradually becomes apparent, and finally results in macroscopic inhomogeneity. When $U / H_{0}>0.15$, the necking is evidently developed. Therefore the initiation of necking occurs between 0.10 and 0.15 . For case 3 , there is more diffuse necking. Several coarsely slipping bands accompany the necking development. Though the distributions of $\tau_{0}^{(i)}$ for five cases are different, the deformation patterns are similar. Comparison of case 1 , case 4 and case 5 , it is found that the level of material property inhomogeneity does not affect the overall deformation patterns. The strain at initiation of necking is insensitive to the level. As the level decreases, the 'patchy' deformation gradually becomes less pronounced. Comparison of case 1, case 2 and case 3 , it is also found that the distribution of CRSS has an influence on the site of necking (case 1 is at lower end and case 2 at upper end) and necking feature (case 3). The plastic deformation patterns are insensitive to the initial material imperfection at deformation $U / H_{0}<0.1$. As the deformation increases, the insensitivity gradually decreases, and eventually results in macroscopic inhomogeneity of plastic deformation. 
It is interesting to compare figure 8-figure 12 with figure 13 where the curves give the load-extension plot for the crystals in different cases. The load $P$ is given by

$$
P=\int_{x_{i}}^{x_{\mathrm{e}}} T_{y}\left(y_{\mathrm{e}}, x\right) \mathrm{d} x
$$

where $x_{\mathrm{i}}$ is left side $x$-coordinate at loading-end, $x_{\mathrm{e}}$ and $y_{\mathrm{e}}$ are the right side $x$ - and $y$ coordinates at loading-end.

From figure 13 , it seems that the maximum load is at $U / H_{0}=0.10-0.12$. The variation of cross contraction at necking with end-displacement is shown in figure 14 for case 1 and case 2. It can be classified into two linear variation stages. The 'knee' of the curves approximately corresponds to the initiation of necking and also to the maxima of loading. It seems that this kind of material inhomogeneity does not modify the overall features of deformation patterns. The differences induced by the inhomogeneity, such as yield stress, shape of load-extension curve and maxima of loading, are common phenomena which can also be observed in experiment.

The corresponding contour plots of slip activities for case 1, case 2 and case 3 are shown in figure 15(a)-(e)-figure 20(a)-(e). From these contour plots, the discrete deformation patterns and discrete slip bands are clearly observed. For case 1, comparing with the CRSS as shown in figure $7(a)$, the CRSS at ISS bands of 8-16 and 28-32 is lower, and that of 2SS in bands from 6-12 and 30-38 is lower. The bands with the lower values of CRSS correspond to the easily slipping bands (figure $15(a)$ and $(b)$ and figure $16(a)$ and $(b)$ ). For case 2, the CRSS at 1SS bands of 11, 10,15-17, 27-30, 38-40, and at 2SS bands of 8, 10-11, $15-16,23,28-29,36-37,40$ are lower (figure $7(b)$ ). These bands are easily slipping bands (figure $17(a)$ and $(b)$ and figure $18(a)$ and $(b)$ ). For case 3 , the CRSS at 1 SS bands of $8-10$, $16-20,34-35$ and at $2 S S$ bands of $2,4,7,11,20,24,28-29$ are lower (figure $7(c)$ ), the corresponding bands also show easily slipping bands (figure $19(a)$ and $(b)$ and figure $20(a)$ and $(b)$ ). For case 4 and case 5, similar phenomena can also be observed (figures 7(e) and (d), figure 11 and figure 12). As loading proceeds the deformation pattern becomes localized in some bands which correspond to a package of contiguous bands with lower CRSS. With continued straining, the localized deformation bands develop into localized zones which may be called CSBs (figure $15(b)$ and (c)-figure 20(b) and (c)). The CSBs may result in the formation of LSBs and necking. Figure 15(c)-(e)-figure 20(c)-(e) show developments of necking and formation of LSBS. The similar results can also be obtained for case 4 and case 5. The contour plots of 2SS for case 4 in figure $21(a)-(c)$ clearly exhibit the development of LSBs. It does not involve a single band, but generally consists of several LSBS. It is also shown that the LSBS are misaligned with slip bands (dashed line). This can also be observed from experiment results (figure 2). The 3SS for all five cases is only active after necking and focuses in small regions.

The experiments show that the CSBs play an important role in the formation of localized bands and necking. From our calculated results, it can be seen that the lower CRSS bands exhibit easily slipping bands. The closely-packed lower CRSS bands may induce the CSBS. CSBS may serve as imperfections to promote the necking and formation of LSBS; this is consistent with experimental results [11]. The calculated results also show that the initial inhomogeneity of material property results in the heterogeneity of plastic deformation which associated with the patchy slip. The patchy slip zones evidently exist in deformed meshes. The patchy slip may also be promoted by the latent hardening effects. In present calculation, $q=1.4$.

The lattice rotation can be seen from figures $8-12$, which shows very inhomogeneous. The inhomogeneous rotation may result in geometrical softening and hardening in crystal. 
The softening promotes the slip activity, and the hardening prohibits the slip activity. This inhomogeneous rotation also results in the curved slip bands at neck. This is consistent with the experimental results as shown in figure $5(b)$. A geometrical softening due to the rotation near free surface at neck is observed in both experimental and our calculated results. The calculated results indicate that the geometrical hardening occurs in the middle of the neck. The experimental result shown in figure 2 confirms the calculated results. Our results also demonstrate that the curved slip bands depend on the amount of conjugate slip therein. The conjugate slip activity may promote the curving of the slip bands. It is also shown that the most inhomogeneous strains locate at neck and the highest slip strain is at the specimen's center, not at the specimen's free surface.

It has been known that the LSBS usually develop from the CSBS and initialize at the free surface. The CSBs at free surface may serve as preferential position to induce the formation of LSBs. Thus the length scale of the fluctuation distributions has an apparent relationship with the formation of the CSBS and LSBs. The width of CSB usually contains several contiguous slip bands with lower CRSS, so the width of LSB may be at same magnitude.

The experiments show that the LSBS on one hand may exhibit a straight mode, and on the other hand, may exhibit a curved mode. In present calculation, we conclude that when LSBS form before necking, they are straight and aligned with the CSBs. When the LSBS form just after necking, the LSBS are also straight but misaligned with CSBs. When necking continues developing after the formation of LSBS, the LSBS become curved and misaligned with the CSBS. These three forms of LSBS are schematically shown in figure $22(a)-(c)$. The experimental results [11] confirm our present calculation. The experiment [11] also shows that formations of CSBs do not involve the nucleation of voids, but the formations of LSBS do. This means that the non-crystallographic effect exists in the formation of LSBs, but not in the formation of CSBS. Our calculated results show that, in the first mode, the formation of LSBS is a crystallographic process, but for the other two modes, the non-crystallographic effect is involved in formation of LSBS. If the LSBS form after necking, the slip lines already become curved. Generally, the LSBS just forming exhibit straight fashion, thus it is impossible for LSBS to be aligned with the curved slip lines.

The straining and microstructure induced anisotropy can be observed in figures 8-12 and figures 15-20. As the deformation proceeds, the initial single crystal becomes 'patchy' and is divided into many zones with different crystal orientation, called subgrain. This kind of deformation pattern can also be found in experiment. The subgrains result from the inhomogeneity of lattice rotation.

\section{Conclusions}

Our rate-dependent finite element calculations based on the DSM show the vital role of nonuniformity of material property on inhomogeneous deformation. The following conclusions are drawn:

(1) The DSM which describes the initial non-uniformity of material property for dislocation moving reasonably reflects the micromechanism in plastic deformation inhomogeneity. But the determination of CRSS distribution from the experiment should be accentuated.

(2) The reduction of cross contraction at neck as a function of loading can be classified into two linear change stages. The 'knee' or the transition of the two stages approximately corresponds to maxima of loading and also corresponds to the initiation of necking.

(3) The bands with lower CRSS are the easily slipping bands. A group of contiguous bands with lower CRSS may result in CSBS which may induce the formation of LSBS and 
necking. The deformation patterns are insensitive to the level of CRSS. The distribution of CRSS may change the site of necking and feature of necking.

(4) When LSBs form before necking, they exhibit a linear fashion and the shearing failure is along certain crystallographic planes. When the LSBs form just after necking and immediately result in shearing failure, the LSBs are also straight but misaligned with CSBs. When the LSBS form after a well-developed diffuse necking and develop after formation, the LSBS are curved and misaligned with the CSBs. The latter two shearing failures involve the non-crystallographic effect. These results are consistent with the experimental results.

(5) The 'patchy' deformation patterns are observed in present calculation. The deformation is related to the inhomogeneity of material property and also related with the high latent hardening rate. Subgrains form due to the 'patchy' deformation in single crystal.

\section{Acknowledgments}

The support of the National Natural Science Foundation of China is gratefully acknowledged. The authors would like to thank the reviewers of the present paper for their very useful comments.

\section{References}

[1] Peirce D, Asaro R J and Needleman A 1982 Acta Metall. 301087

[2] Peirce D, Asaro R J and Needleman A 1983 Acta Metall. 311951

[3] Nemat-Nasser S 1992 Appl. Mech. Rev. 45 S19

[4] Neuhauser H 1988 Solid State Phenomena 3\&4 407

[5] Mader S 1957 Z. Phys. 14973

[6] Zhang Y W 1992 PhD Thesis Northwestern Polytechnical University

[7] Hill R 1962 J. Mech. Phys. Solids 101

[8] Asaro R J and Rice J R 1977 J. Mech. Phys. Solids 25309

[9] Needieman A and Tvergaard V 1992 Appl. Mech. Rev. 45 S3

[10] Needleman A 1972 J. Mech. Phys. Solids 20111

[11] Chang Y W and Asaro R J 1981 Acta Metall. 29229

[12] Elam C F 1927 Proc. $R$ Soc. Lond. A115 133

[13] Piercy G R, Cahn R W and Cottrell A H 1955 Acta Metall. 3331

[14] Kafka V 1985 Czech. J. Phys, B 35346

[15] Taylor G I 1938 J. Inst. Metals 62307

[16] A.saro R J 1983 Adv. Appl. Mech. 23 I

[17] Hill R and Rice J R 1972 J. Mech. Phys. Solids 20401

[18] Needleman A, Asaro R J, Lemonds J and Peirce D 1985 Comp. Meth. Appl. Mech. Eng. 52689

[19] Asaro R J and Needleman A 1985 Acta Metall. 33923

[20] Rice J R 1987 Mechanics of Materials 6317

[21] Mohan R, Ortiz M and Shih C F 1992 J. Mech. Phys. Solids 40315

[22] Staker M R and Holt D L 1972 Acta Metall. 20569

[23] Harren V, Lowe T C, Asaro R J and Needleman A 1989 Phil. Trans. R. Soc. Lond. A 328443 